\title{
AN INFINITE CONSTRUCTION IN RING THEORY
}

\author{
by E. A. WHELAN
}

(Received 15 June, 1987; revised 9 September, 1987)

1. Introduction. In this note we describe a class of functors on the category of associative rings with unity (hereafter "rings") and of ring homomorphisms which, loosely speaking, 'preserve the properties' of two-sided ideals, but can be chosen to be arbitrarily 'bad' for one-sided properties of rings. Our main result is:

THEOREM 1.1. Let $t$ be any cardinal number. There exists a functor $G_{t}(-)$ on the category of rings to itself such that the ring $G=G_{t}(R)$ has the following properties:

(1) it is a ring extension of $R$, and is generated as $R-R$ bimodule by elements $\left\{E_{I J}\right\}$ (where the indices $I, J$ are explained in Section 2) centralising $R$, such that $1_{R} \in\left\{E_{I J}\right\}$, and $\left\{E_{I J}\right\}=\left\{1_{R}\right\}$ if $t=0$ while $\left\{E_{I J}\right\}$ is infinite if $t>0$;

(2) the set $\left\{E_{I J}\right\} \cup\{0\}$ is multiplicatively closed;

(3) $G$ is left free and right free as $R$-module on $\left\{E_{I J}\right\}$;

(4) if $t \neq 1$ every two-sided ideal (hereafter simply "ideal") of $G$ is an extended ideal, generated (as an ideal of $G$ ) by its intersection with $R$;

(5) distinct ideals of $R$ have distinct extensions in $G$;

(6) if $t \neq 1$, extension and contraction of ideals preserve sums, intersections and multiplication;

(7) if $t>1, G$ has infinite direct sums of one-sided ideals on both sides, and infinite ascending and descending chains of left and of right annihilator ideals;

(8) if $t>1$ then every finitely generated one-sided G-module is cyclic.

We call these functors $G$-functors, and shall say that the case $t=0$ is the trivial case, the case $t=1$ is the singular case, and that the cases $t>1$ are the non-trivial cases.

In Section 2 we go through the basic construction for $G$-functors, and establish the properties listed above. In Section 3 we investigate further properties of the ring extension $R \rightarrow G_{t}(R)$ : the behaviour of maximal, primitive and prime ideals, the properties of one-sided $G$-modules and one-sided ideals of $G$, the centre of $G$, rings of quotients of $G$, and 'canonical' generation of two-sided ideals of $R$ and of $G$. We show that the non-trivial $G$-functors can be used to construct arbitrarily large classes of pairwise non-isomorphic, non-noetherian rings in which two-sided ideals are generated in various 'canonical' ways. The results in $[14,15,16,17,18]$ can then be used to show that in appropriate cases the rings obtained have 'good' properties (e.g., for a suitable choice of $R$, they' satisfy the d.c.c. on prime ideals, or a suitable version of the 'principal ideal theorem' or of the Jacobson conjecture, or they have a good notion of unique factorisation), despite behaving very 'badly' in respect of one-sided properties.

In Section 4 we use $G$-functors to show that rings in which (selected) ideals are left-right principal can be very badly behaved, by contrast with rings in which the analogous ideals are generated by normalising elements. But in Section 5 we show that

Glasgow Math. J. 30 (1988) 349-357. 
both the Jacobson conjecture and the problem of d.c.c. on prime ideals for left-right noetherian rings can be reduced to related problems about such rings, so they are likely to repay further study.

I would like to thank A. W. Chatters, T. H. Lenagan, J. C. Robson and the referee for critical comments on earlier expositions of this construction. All unexplained notation and terminology are entirely standard, and proofs are generally sketched or omitted.

2. The Basic Construction. Let $t$ be a cardinal number, and assume that all rings are non-zero. A $G$-construction pair is an ordered pair $\left\langle B, T_{1}\right\rangle$ where $B$ is an infinite set of cardinality $b \geqslant t=\operatorname{card}\left(T_{1}\right)$ and $T_{1}$ is a set of injective mappings $s: B \rightarrow B$ which satisfies the conditions (a) $s \in T_{1}$ implies card $(B \backslash \operatorname{Im}(s))=b$, and (b) $s, w \in T_{1}$ and $s \neq w$ implies $\operatorname{Im}(s) \cap \operatorname{Im}(w)=\varnothing$.

If $\left\langle B, T_{1}\right\rangle$ is a $G$-construction pair we let $H_{1}$ be the unital monoid of injective mappings generated by $T_{1}$ (so that $i d_{B} \in H_{1}$ ), and $H_{2}=\left\{\operatorname{Im}(s): s \in H_{1}\right\}$. Similarly, we let $T_{2}=\left\{\operatorname{Im}(s): s \in T_{1}\right\}$. Note that $t$ may take any value from 0 to $b$, and that, for $t>1, H_{2}$ has the properties (i) $I \in H_{2}$ and $I \neq B$ implies there exists $J \in H_{2}$ such that $I \cap J=\varnothing$, and (ii) $I, J \in H_{2}$ implies $I \cap J=\varnothing$ or $I \subseteq J$ or $J \subseteq I$.

If $t>1$ then it is possible to choose $T_{1}$ such that $T_{2}$ is a set of equivalence classes in $B$. This is impossible in both the trivial case (where $T_{2}=\varnothing$ ) and in the singular case (where $T_{2}$ is a singleton). Similarly, property (i) is not satisfied in the singular case, and is only satisfied vacuously in the trivial case.

Now let $M$ be the bimodule direct sum of $b=\operatorname{card}(B)$ copies of $R$ (with the usual bimodule structure), and for each $i \in B$ let $x_{i}$ be the element of $M$ with 1 in the $i$ th place and 0 elsewhere. For $I, J \in H_{2}$ let $E_{I J}$ be the bimodule endomorphism of $M$ (written on the left) defined by:

$$
\begin{array}{ll}
E_{I J}\left(x_{i}\right)=0 & (i \notin J), \\
e_{J J}\left(x_{i}\right)=x_{s w^{-1}(i)} & (i \in J)
\end{array}
$$

where $s, w \in H_{1}$ and $I=\operatorname{Im}(s), J=\operatorname{Im}(w)$. Let $G_{t}(R)$ be the subring of $\operatorname{End}\left(M_{R}\right)$ generated by $\left\{E_{l J}\right\}$ and the left multiplications by elements of $R$. Identify $r \in R$ with left multiplication by $r$, so that $R$ is identified with a subring of $G_{t}(R)$.

Proposition 2.1. If $I, J, K, L \in H_{2}$ and $p, q, w, s \in H_{1}$ are such that $I=\operatorname{Im}(p)$, $J=\operatorname{Im}(q), K=\operatorname{Im}(w)$ and $L=\operatorname{Im}(s)$, then:

$$
\begin{array}{ll}
E_{I J} E_{K L}=0 & \text { if } J \cap K=\varnothing ; \\
E_{I J} E_{K L}=E_{P Q} & \text { if } J \cap K \neq \varnothing,
\end{array}
$$

where in this case $P=p q^{-1}(J \cap K)$ and $Q=s w^{-1}(J \cap K)$.

Proof. This is a straightforward though tedious matter of verification.

COROLlaRY 2.2. With the same conventions:

(a) (closure) $\left\{E_{I J}\right\} \cup\{0\}$ is a multiplicatively closed subset of $G=G_{t}(R)$;

(b) (identity) $E_{B B}=1_{R}=1_{G}$; 
(c) (factors of identity) if $I, J \in H_{2}$ then $1_{G}=1_{R}=E_{B I} E_{I J} E_{J B}$, so that each element of $\left\{E_{I J}\right\}$ generates $G$ as ideal of $G$;

(d) (cancellation) if $I, J, K, L, P, Q \in H_{2}$ then $E_{I J} E_{K L}=E_{P Q} E_{K L} \neq 0$ implies $I=P$ and $J=Q$.

This proves (2) of Theorem 1.1.

Proposition 2.3. The ring $G$ is an extension of $R$, freely generated as left and as right $R$-module by $\left\{E_{I J}\right\}$.

Proof. The trivial case is obvious, so consider the non-trivial cases. Clearly for fixed $I^{\prime}, J^{\prime}$ each $R E_{I^{\prime} J^{\prime}}$ is left free on $E_{I^{\prime} J^{\prime}}$, so if $G$ is not left free over $\left\{E_{I J}\right\}$ then there is a least integer $d \geqslant 2$ such that there is a (left) dependence relation

$$
a_{1} E_{I(1) J(1)}+\ldots+a_{d} E_{I(d) J(d)}=0
$$

with distinct $E_{I(i) J(i)}$ and each $0 \neq a_{i} \in R$. If $J(1)=J(2)=\ldots=J(d)$ then $I(i) \neq I(j)$ for some $i, j, 1 \leqslant i, j \leqslant d$, and then we can find $E_{K L}$ such that

$$
E_{K L} E_{I(i) J(i)} \neq 0=E_{K L} E_{I(j) J(j)},
$$

(or the alternative with $=$ and $\neq$ interchanged). From Corollary 2.2 (d) (cancellation), this contradicts the minimality of $d$. The case in which $J(k) \neq J(r)$ for some $k, r, 1 \leqslant k$, $r \leqslant d$ is similar. The singular case (i.e., $t=1$ ) then follows from the obvious fact that, if $t(1), t(2)$ are cardinals and $t(1) \leqslant t(2)$ then there is an embedding $G_{t(1)}(R) \rightarrow G_{t(2)}(R)$ of ring extensions of $R$ (induced by any embedding of the corresponding sets $T_{1}$ ).

This proves (1) and (3) of Theorem 1.1.

Proposition 2.4. If $x \in G$ in a non-trivial case, and if $x=\sum a_{I J} E_{I J}$ (with $a_{I J} \in R$ and $a_{I J}=0$ except possibly for finitely many elements $E_{I J}$ ) then $G x G=\sum G a_{I J} G=\sum A E_{I J}$, where $A$ is the ideal of $R$ generated by $\left\{a_{I J}\right\}$.

Proof. This is easily seen by 'killing off' all but a selected one of those $E_{I J}$ for which $a_{I J} \neq 0$, using the orthogonality relations between the elements of $\left\{E_{I J}\right\}$.

This proves (4), (5) and (6) of Theorem 1.1 (since in the trivial case we have simply $R=G$, and (4) is obviously true for $t=1$ ). Point (7) of Theorem 1.1 then follows from the multiplication rules in $\left\{E_{I J}\right\} \cup\{0\}$ (Proposition 2.1).

Proposition 2.5. If $t \geqslant 1$, every finitely generated one-sided $G$-module is cyclic.

Proof. Let $A$ be a right $G$-module, and suppose $y_{1}, \ldots, y_{n} \in A$. We can find $J(1), \ldots, J(n) \in H_{2}$ such that $1 \leqslant i, j \leqslant n$ and $i \neq j$ implies $J(i) \cap J(j)=\varnothing$. It follows that $\sum y_{i} G=y G$, where $y=\sum y_{i} E_{B J(i)}$.

This proves point (8) of Theorem 1.1.

It is clear that each $G_{t}(-)$ is a functor in the obvious way, and it is easy to check that, if $G_{t}(-)^{*}$ is the corresponding functor for a given value of $t$, but with different choices in 
the construction, then $G_{t}(-)$ and $G_{t}(-)^{*}$ are naturally equivalent functors. Theorem 1.1 is completely proved.

The results about contraction of ideals that are proved above do not extend to the singular case $t=1$, as the following example shows.

EXAmpLE 2.6. Suppose that $F$ is the field with two elements, and that $S=G_{1}(F)$. If $B \neq X \in H_{2}$ then a counting argument shows that the element $1+E_{X X}$ of $S$ does not generate the unit or the zero ideal, even though $F$ is a simple ring. Hence $1+E_{X X}$ does not generate the unit ideal in $G_{1}(\mathbb{Z})$.

3. Properties of G-functors. Canonical generation of ideals. In our work in $[14,15,16,17,19]$ we have been concerned with rings in which two-sided ideals were generated in various canonical ways, but in which no properties of one-sided ideals were assumed. Consider the following properties that a ring $R$ might have:

(a) every ideal is generated by a central element;

(b) every ideal is generated by a normal (ising) element, i.e. an element $u$ such that $R u=u R$ (a quasi-commutative principal ideal ring, or QCPIR [17]);

(c) every ideal is right finitely generated;

(c) ${ }^{\mathrm{s}}$ every ideal is left-right finitely generated;

(d) every ideal is right principal (so that $R$ is an ideal principal right ideal ring, or IPRI-ring [11]);

(d) ${ }^{s}$ every ideal is left-right principal (so that ([11]) $R$ is an IPLI-IPRI ring);

(e) every ideal is generated by a finite sequence $c_{1}, \ldots, c_{n}$ such that $c_{1}$ is central in $R, c_{2}+\left(R / c_{1} R\right)$ is central in $R / c_{1} R$, and so on;

(f) every ideal is generated by a finite sequence $c_{1}, \ldots, c_{n}$ such that $c_{1}$ is normalising in $R, c_{2}+\left(R / c_{1} R\right)$ is normalising in $R / c_{1} R$, and so on;

(In $[15,16]$ we call the sequences discussed at (e) and (f) a finite sub-centralising sequence and a finite sub-normalising sequence respectively, though other terminologies are widely used. A ring with the property (f) is called a bi-noetherian pseudo-commutative ring, or $B P C$ ring.)

(g) every non-minimal prime ideal of $R$ contains a non-minimal prime generated by a normalising element. (Subject to assorted extra conditions, such rings are known in the literature as unique factorisation rings, or UFRs; we shall use the same term without assuming any other conditions.)

THEOREM 3.1. Each of the properties $(a)$ to $(g)$ above is preserved by a non-singular ring extension $R \rightarrow G_{t}(R)$.

Moreover, properties (c), (d) and properties (c) $)^{\mathrm{s}},(\mathrm{d})^{\mathrm{s}}$ are equivalent pairs for $G_{t}(R)$ in the non-trivial cases (from Proposition 2.5), and it is easy to check that they then imply (c) and (c) (but not necessarily (d) or (d) $)^{\mathrm{s}}$ ) for $R$. Properties (a) and (e) also 'contract' from $G_{t}(R)$ to $R$, but we do not know whether (and doubt that) properties (b) or (f) do. Property $(\mathrm{g})$ (being a UFR) in the case of left-right noetherian rings has been discussed by Chatters and Jordan $[3,2]$, and by other writers $[5,9]$, and is discussed in a more 
general context in [19]; it is clearly preserved by a non-singular ring extension $R \rightarrow G_{t}(R)$, but we do not know whether (and doubt that) it contracts from $G_{t}(R)$ to $R$.

It is an extremely difficult problem, given rings $R, S$ and cardinals $a, b>1$ to determine whether $G_{a}(R)$ and $G_{b}(S)$ are isomorphic rings. But in certain cases non-isomorphism can be easily shown, as follows. Let $C$ be a maximal ideal of the centre $K$ of a ring $R$, chosen so that $d=\operatorname{dim}_{K / C}(R / R C)$ is maximal. If $a, b>d$ are infinite cardinals then $G_{a}(R) \simeq G_{b}(R)$ implies $a=b$, by dimensionality. Hence, if $R$ is commutative, the rings $G_{a}(R), G_{b}(R)$ are non-isomorphic when $a \neq b$ are infinite, so the functors $G_{a}(-), G_{b}(-)$ are non-equivalent.

The following result is then immediate.

THEOREM 3.2. If $R$ is a ring satisfying one of conditions $(a)$ to $(g)$, then the class of rings $\left\{G_{t}(R)\right\}$ contains any arbitrarily large (cardinal) number of pairwise non-isomorphic non-noetherian rings satisfying the same condition.

Paraphrasing, 'almost all' QCPIRs, BPC rings and UFRs are non-noetherian.

There are some other features of the non-trivial cases which are worth noting.

\section{Separability}

(i) It is easy to check that the centraliser in $G$ of $\left\{E_{I J}\right\}$ is $R$, and hence that the centre of $G$ is the centre of $R$. Thus if $R$ is commutative then the properties of the ring extension $R \rightarrow G$ resemble those of a separable algebra (see [4] and references therein). But, since $t>1, G$ is not a separable $R$-algebra, because it is $R$-projective, and if separable as an algebra would be finitely generated as an $R$-module, by the theorem of Villamayor and Zelinsky [4, p. 47]. Specifically, $G$ is a separable ring in the sense of Azumaya [1] if and only if $R$ is also a separable ring, which is true when $R$ is commutative.

(ii) In the same vein, work has been done in the non-commutative case by $\mathrm{K}$. Sugano, K. Hirata and others on $H$-separable ring extensions (see [13] and references therein), which resemble separable extensions of commutative rings. For a not-necessarily commutative ring $R$, the ring extension $R \rightarrow G$ has properties which resemble those of an $H$-separable extension.

\section{One-sided ideals}

(iii) If $A$ is a right ideal of $R$ then $A G=\sum E_{I J} A$, so that if $C$ is an ideal of $R$ then $G C G=G C=C G$.

(iv) If $K$ is the right $R$-submodule $\sum_{I \neq B} E_{I J} R$ of $G$ then $K$ is a right ideal of $G$, and the correspondence $N \leftrightarrow(N G+K) / K$ gives a lattice isomorphism between the right ideals of $R$ and the right $G$-submodules of $G / K$. This lattice isomorphism respects multiplication by (corresponding) two-sided ideals of $R$ and of $G$. Hence the extension of a (right) primitive ideal of $R$ is a (right) primitive ideal of $G$, and $J(G) \subseteq G J(R) G$. 


\section{Rings of fractions}

(v) If $R$ is any ring then the regular elements of $R$ are regular in $G$, so that if $R$ is semiprime right Goldie then the set $C(0)$ of regular elements of $R$ is a right Ore set of regular elements in $G$. Hence $G$ has a right ring of quotients $Q$ with respect to $C(0)$, and $Q$ is a finite direct sum of non-noetherian (in fact, non-Goldie) simple rings. Similarly (see [14]), if $R$ is semiprime of finite bimodule uniform dimension, and if every non-zero ideal of $R$ contains a non-zero normalising element, then $G$ has a similar left-right ring of quotients $W$ which is a finite direct sum of non-Goldie simple rings.

\section{Radicals}

(vi) In [19] we define an ideal $Q$ of a 'ring not necessarily with unity' $R$ to be quasi-primitive if $Q$ is prime and $R / Q$ is subdirectly irreducible. If $M, Q$ and $P$ are respectively a maximal, a quasi-primitive and a prime ideal of $R$ then their extensions in $G$ are respectively maximal, quasi-primitive and prime. The corresponding statements about contracted ideals are also true, from point (6) of Theorem 1.1. It follows that the Brown-McCoy and Prime radicals and the intersection of all the quasi-primitive ideals are each preserved both by the extension $R \rightarrow G$, and by contraction from $G$ to $R$. We show in Section 5 that the corresponding statements about primitive and semi-primitive ideals are false; the contraction of a (right) primitive ideal of $G$ need not be primitive, and the inclusion $J(G) \subseteq G J(R) G$ can be strict.

\section{Goldie conditions}

(vii) For any ring $R, G=G_{t}(R)$ may be chosen to be arbitrarily 'bad' for one-sided ideals in the following sense: if $t$ is infinite, $G$ not only contains infinite ascending and descending chains of annihilator ideals on either side, it also contains infinite direct sums of $t$ non-zero right (or left) ideals.

4. IPLI-IPRI Rings. If $R$ is a left-right noetherian ring or a BPC ring then any non-trivial $G=G_{t}(R)$ is an IPLI-IPRI ring (though certainly non-noetherian!). Subject to some one-sided chain conditions, IPRI or IPLI-IPRI rings are known to be quite well-behaved [11, 7], as are rings in which selected ideals are generated by normalising elements. By contrast, IPLI-IPRI rings without one-sided chains conditions can be very 'bad' indeed.

ExAmples 4.1. (a) If $R$ is a ring in which every ideal is generated by a normalising element (a QCPIR) then every prime ideal $P$ of $R$ is of height $\leqslant 1$ [17].

(b) Similarly, if $S$ is a 'ring not necessarily with unity' and $u \in S$ satisfies $S=S u=u S$ then $S$ does have a unity ([15], Chapter 8).

Neither analogue of these statements need to be true if we only assume that ideals are (multiplicatively) left and right principal.

Counter-examples 4.2.

(a) In [10] Nagata shows that there exists a commutative noetherian unique factorisation domain $D$ with prime ideals of arbitrarily large height, so that the ring $D$ has 
infinite 'altitude'. If $t>1$ then in $G=G_{t}(D)$ every ideal is left-right principal but prime ideals can still have arbitrarily large height. This ring is a BPC ring.

(b) Similarly, in [12] Robson exhibits a noetherian domain $E$ with exactly three ideals. If $K$ is the only proper ideal, and if $H$ is the corresponding ideal of $G_{t}(E)$ then $H$ has no unity element, but is a simple, idempotent 'ring not necessarily with unity' containing elements $a, b$ such that $H=H a=b H$.

5. Semiprimitivity. Unlike the radicals previously mentioned, the Jacobson radical does not behave well under $G$-functors. Unless otherwise specified, we assume for this Section that all $G$-functors are non-trivial. It is then easy to verify

Proposition 5.1. If $G=G_{t}(R)$ is a non-trivial $G$-functor, and $a$ is a right regular non-unit of $R$, then, for $J \neq B$, the element $1+a E_{B J}$ of $G$ has no right quasi-inverse.

Corollary 5.2. Suppose that $R$ is a commutative local non-simple domain, with maximal ideal $M=J(R)$. Then $G M G$ is the Brown-McCoy radical of $G$, but $J(G)=0$.

What is happening in this situation is that the 'extra' non-commutativity of $G$ ensures that, for 'reasonable' $R, J\left(G_{t}(R)\right)$ is strictly contained in the extension of the Jacobson radical of $R$. Essentially the same argument establishes the following.

Proposition 5.3. If $R$ is commutative or one-sided notherian or BPC or IPLI, the Jacobson radical of $G$ is the prime radical of $G$, i.e. the extension of the prime radical of $R$, and is nilpotent if $R$ satisfies a.c.c. on two-sided ideals

Nevertheless, consideration of selected bimodules over arbitrary rings suggests that the study of IPLI-IPRI rings might (via $G$-functors) clarify several outstanding problems in the theory of noetherian rings. In several papers (see [8] and references therein) Jategaonkar, Brown, Stafford and other writers have studied bimodules ${ }_{S} B_{R}$ over rings $S$, $R$ subject to the assumptions that $S, R$ are left (respectively, right) or two-sided noetherian, and $B$ is left (respectively right) noetherian as $S$ - (respectively $R$-) module. An alternative approach is to restrict attention to idealistic bimodules defined in ([15], Chapter 8) as $R-R$ bimodules (for a single ring $R$ ), of the form $A / B$ for ideals $B \subseteq A$ of $R$. This gives a strictly smaller class of bimodules, even when $R=S$, as is easily seen from the example of a commutative noetherian ring with a non-trivial automorphism.

In [15] we defined the left and right $K$-radicals of $R$ as the sets of elements which left (respectively, right) annihilate all simple idealistic bimodules, and denoted them by ${ }^{*} K(R)$ and $K^{*}(R)$ respectively. The $K$-radical is then defined as $K(R)={ }^{*} K(R) \cap K^{*}(R)$, and is the set of elements which annihilate all simple idealistic bimodules from both sides.

In any ring, each of these radicals is an ideal, with the usual properties under epimorphisms. There are examples of rings in which the left and right $K$-radicals are different, and in which there are various inclusion relations between them and the Jacobson and Brown-McCoy radicals ([15], Chapter 8). The very nice thing about the 
$K$-radicals is that each $K$-radical is clearly preserved both under extension and under contraction by any non-singular $G$-functor, quite unlike the case of the Jacobson radical.

It can be shown ([15], Chapter 5, or [16]) that in a bi-noetherian pseudocommutative ring $R$ the left and right $K$-radicals, and therefore the $K$-radical, are both equal to the Brown-McCoy radical $H=H(R)$, and that $\bigcap_{i=1}^{\infty} H^{i}=0$ (so that $\bigcap_{i=1}^{\infty} J(R)^{i}=0$ too).

For any ring $R$, the Jacobson radical $J=J(R)$ is contained in $H(R)$, and if every ideal of $R$ is left (right) finitely generated then $J \subseteq{ }^{*} K(R)\left(J \subseteq K^{*}(R)\right.$ respectively). Thus if every ideal is left-right finitely generated, for example if $R$ is a IPLI-IPRI ring, we have $J \subseteq K(R)$, so the intersection theorem for $K(R)$ would imply the Jacobson conjecture for $R$ (as in the case of BPC rings).

This matters because of the emphasis in recent literature (see [8]) on the subdirectly irreducible factor rings of a ring $R$. The connection is brought out by the following result.

Proposition 5.4. Let $R$ be a ring. Then an $R-R$ bimodule $M$ is isomorphic to an idealistic bimodule $A / B$ if and only if $M$ is bimodule isomorphic to an essential ideal $N$ of a factor ring $R / C$ of $R$. Moreover, $M$ is simple idealistic if and only if it is bimodule isomorphic to the heart $B$ of a subdirectly irreducible factor ring of $R$.

Proof. One way round is trivial, so suppose $A / B$ is an idealistic bimodule. Let $C$ be an ideal of $R$ such that $B \subseteq C$ and $C / B$ is a complement ideal for $A / B$ in $R / B$. Then

$$
N=(A+C) / C \simeq A /(A \cap C)=A / B
$$

and $N$ is an essential ideal of $R / C$. The final assertion is then obvious.

The significance of this result is that it characterises the three $K$-radicals in terms of the hearts of the subdirectly irreducible factor rings of $R$. Using ([6], Lemma 5.2) it is easy to show that, for a left-right noetherian ring $R,{ }^{*} K(R)=K^{*}(R)=K(R)=\cap Q$, where $Q$ ranges over the quasi-primitive ideals of $R$. These observations prompt the following conjectures, which are true in all cases we know of, and the second of which would imply the Jacobson conjecture for left-right noetherian rings.

CONJECTURE 5.5. In an IPLI-IPRI ring $R$, the left and right $K$-radicals coincide, and are equal to the intersection of all the quasi-primitive ideals of $R$.

CONJECTURE 5.6. In an IPLI-IPRI ring $R$, the $K$-radical $K=K(R)$ satisfies the intersection theorem, that is:

$$
\bigcap_{i=1}^{\infty} K^{i}=0
$$

Similarly, the d.c.c. for prime ideals of an IPLI-IPRI ring would imply the d.c.c. for prime ideals of a left-right noetherian ring. It is known [14] that the prime ideals of a BPC ring satisfy the d.c.c.. 


\section{REFERENCES}

1. G. Azumaya, Separable rings, J. Algebra 63 (1980), 1-14.

2. A. W. Chatters, Non-commutative unique factorization domains, Math. Proc. Camb. Phil. Soc., 95 (1984), 49-54.

3. A. W. Chatters and D. A. Jordan, Non-commutative unique factorisation rings, J. London Math. Soc. (2) 33 (1986), 22-32.

4. F. Demeyer and E. Ingraham, Separable algebras over commutative rings, Lecture Notes in Mathematics No. 181 Springer-Verlag, 1971).

5. M. P. Gilchrist and M. K. Smith, Noncommutative UFD's are often PID's Math. Proc. Camb. Phil. Soc., 95 (1984), 417-419.

6. A. V. Jategaonkar, Noetherian bimodules, primary decomposition and Jacobson's conjecture, J. Algebra 71 (1981), 379-400.

7. A. V. Jategaonkar, Left principal ideal rings, Lecture Notes in Mathematics No. 123 (Springer-Verlag, 1970).

8. A. V. Jategaonkar, Localization in noetherian rings, London Math. Soc. Lecture Notes Series No. 98, (Cambridge University Press, 1986).

9. L. Le Bruyn, Trace rings of generic matrices are unique factorisation domains, Glasgow Math. J. 28 (1986), 11-13.

10. M. Nagata, Local rings. (Interscience, New York, 1962).

11. J. C. Robson, On PRI and IPRI rings, Quart. J. Math. Oxford (2), 18 (1967), 125-145. 45-81.

12. J. C. Robson, Idealizers and hereditary noetherian prime rings, J. Algebra 22 (1972),

13. K. Sugano, Note on cyclic Galois extensions, Proc. Japan Acad., 57 Ser. A (1981), 60-63.

14. E. A. Whelan, Reduced rank and normalising elements. J. Algebra 113 (1988), 416-429.

15. E. A. Whelan, Thesis (University of East Anglia, 1986).

16. E. A. Whelan, Normalising elements and radicals. Bull. Austral. Math. Soc. Ser. A., to appear.

17. E. A. Whelan, Quasi-commutative principal ideal rings. Quart. J. Math. Oxford (2) 37 (1986), 375-383.

18. E. A. Whelan, Not-necessarily commutative unique factorisation rings, to be submitted to Quart. J. Math. Oxford (2).

19. E. A. Whelan, Radical properties and the Jacobson conjecture, in preparation.

School of Mathematics and Physics

UNIVERSITY OF EAST ANGLIA

NORWICH, NORFOLK NR4 7TJ

ENGLAND 